\title{
Wie belastend ist der Erhalt einer Krebsdiagnose?
}

Hintergrund und Fragestellung: Ist der Erhalt einer Krebsdiagnose mit einer erhöhten Mortalität durch Suizid oder kardiovaskulären Tod assoziiert? Für den Betroffenen stellt der Erhalt einer Krebsdiagnose - insbesondere bei schlechter Prognose eines Tumors - immer eine große Belastung dar. Frühere Studien haben eine Assoziation zwischen einer erhöhten Suizidrate und einer erhöhten Inzidenz kardiovaskulärer Ereignisse bei Krebspatienten gezeigt, diesen Zusammenhang aber eher als Folge der Belastungen durch die Therapie und das Leben mit einem progressiven Leiden angesehen. Die Autoren der vorliegenden Studie untersuchten an einem sehr großen schwedischen Patientenregister die Assoziation von Suiziden und Herztod und einer kürzlich erhaltenen Krebsdiagnose.

Patienten und Methode: In diese retrospektive Kohortenstudie wurden 6.073.240 Fälle eingeschlossen, die zwischen 1991 und 2006 in einem schwedischen Patientenregister erfasst worden waren. Durch Regressionsmodelle und Fall-Kon-

Originalie

Fang $\mathrm{F}$ et al. Suicide and cardio vascular death after a cancer diagnosis. N Engl J Med. 2012 366(14):1310-8. troll-Designs wurde die Assoziation zwischen dem Erhalt einer Krebsdiagnose und Tod durch Suizid oder Herztod im Zeitraum von einer Woche nach Diagnose bis über ein Jahr errechnet. Adjustiert wurde unter anderem für Alter, Geschlecht und sozioökonomischen Status. Die Rolle vorheriger stationärer Aufenthalte wegen psychiatrischer oder kardiovaskulärer Diagnosen wurde durch Abgleich mit dem schwedischen Register stationärer Behandlungsfälle ermittelt.

Ergebnisse: Die Autoren zeigten, dass das relative Suizidrisiko von Patienten, die innerhalb von einer Woche eine Krebsdiagnose erhalten hatten, um das 12,6-fache gegenüber Patienten ohne Krebs erhöht war (95\%-Konfidenzintervall [95\%-KI] 8,617,$8 ; 29$ Suizidopfer), innerhalb des ersten Jahres war die Suizidmortalität immer noch um das 3,1-fache erhöht (95\%-KI 2,73,$5 ; 260$ Suizidopfer). Das Risiko für einen Herztod war um das 5,6- beziehungsweise das 3,3-fache erhöht. Die Risikoerhöhungen fielen ab dem ersten Jahr nach der Diagnose deutlich ab. Die erhöhten Suizid- und Herztodraten fanden sich insbesondere bei Tumoren mit schlechter Prognose und waren nicht durch vorherige stationäre Aufenthalte wegen psychiatrischer oder kardialer Diagnosen erklärbar.

Schlussfolgerung der Autoren: Der Erhalt einer Krebsdiagnose ist mit einer erhöhten Mortalität durch Suizid und Herztod assoziiert, insbesondere bei Krebsformen mit schlechter Prognose.

Schlüsselwörter: Krebsdiagnose - Suizid - Herztod - Mortalität

-Kommentar von Klaus Lieb, Mainz

\section{Spielen auch psychiatrische Diagnosen eine Rolle?}

Der Erhalt einer Krebsdiagnose ist nachvollziehbar ein sehr belastendes Ereignis für die Betroffenen. Gut bekannt ist, dass Krebspatienten eine erhöhte Suizidrate und eine erhöhte Inzidenz kardiovaskulärer Ereignisse zeigen. Neu an der Studie ist, dass die Autoren erstmals eine erhöhte Assoziation zwischen Tod durch Suizid oder Herztod und einer kürzlich erfolgten Krebsdiagnose zeigen konnten. Allerdings muss man beachten, dass die absoluten Suizidraten sehr gering waren. Auf 534.154 Krebsfälle im Beobachtungszeitraum kam es zu 760 Suiziden, von denen sich 29 in der ersten Woche und 260 innerhalb des ersten Jahres nach der Krebsdiagnose ereigneten. Insofern dürfte die praktische und therapeutische Konsequenz eher gering sein. Interessanterweise hatte dieselbe Arbeitsgruppe kürzlich gezeigt, dass die Suizidmortalität nach Erhalt einer Prostatakrebsdiagnose erhöht war. Dies könnte bei einer immer wieder diskutierten Überdiagnostik von Prostatakrebs von Bedeutung sein. Dass die erhöhte Suizidrate nach Krebsdiagnose nicht doch auf psychiatrische Vordiagnosen zurückgeführt werden kann, ist durch die hier vorliegende Studie nicht sicher auszuschließen, da nur stationär behandlungsbedürftige Patienten mit psychiatrischen Diagnosen erfasst wurden. So könnte es sein, dass bei den späteren Suizidopfern schon im Vorfeld ambulant behandelte Depressionen vorlagen, die dann infolge der Belastung durch die Krebsdiagnose im Suizid endeten.

Erstpubliziert in InFo Neurologie und Psychiatrie. 2012;14(6):20.

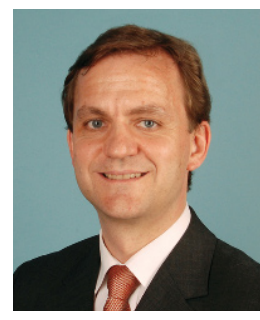

Prof. Dr. Klaus Lieb

Direktor der Klinik für Psychiatrie und Psychotherapie der Universitätsmedizin Mainz, klaus.lieb@unimedizin-mainz.de 